PROCEEDINGS OF THE

AMERICAN MATHEMATICAL SOCIETY

Volume 135, Number 10, October 2007, Pages 3193-3204

S 0002-9939(07)08827-2

Article electronically published on May 14, 2007

\title{
EXTREMAL EXTENSIONS FOR THE SUM OF NONNEGATIVE SELFADJOINT RELATIONS
}

\author{
SEPPO HASSI, ADRIAN SANDOVICI, HENK DE SNOO, AND HENRIK WINKLER
}

(Communicated by Joseph A. Ball)

\begin{abstract}
The sum $A+B$ of two nonnegative selfadjoint relations (multivalued operators) $A$ and $B$ is a nonnegative relation. The class of all extremal extensions of the sum $A+B$ is characterized as products of relations via an auxiliary Hilbert space associated with $A$ and $B$. The so-called form sum extension of $A+B$ is a nonnegative selfadjoint extension, which is constructed via a closed quadratic form associated with $A$ and $B$. Its connection to the class of extremal extensions is investigated and a criterion for its extremality is established, involving a nontrivial dependence on $A$ and $B$.
\end{abstract}

\section{INTRODUCTION}

Let $A$ and $B$ be nonnegative selfadjoint relations (multi-valued operators) in a Hilbert space $\mathfrak{H}$. Their operator-like sum $A+B$, defined as

$$
A+B=\{\{f, g+h\}:\{f, g\} \in A,\{f, h\} \in B\},
$$

is a nonnegative relation in $\mathfrak{H}$. Here $\operatorname{dom}(A+B)=\operatorname{dom} A \cap \operatorname{dom} B$ is not necessarily dense in $\mathfrak{H}$, even in the case that $A$ and $B$ are densely defined operators. Furthermore, (the closure of) $A+B$ need not be an operator; consequently all selfadjoint extensions of $A+B$ can be multi-valued. Standard results concerning (nonnegative) selfadjoint extensions of closed symmetric operators do not directly apply for studying selfadjoint extensions of $A+B$. The recent factorization approach developed in its most general setting in $[9$ turns out to be particularly useful for studying nonnegative selfadjoint extensions of $A+B$. The idea is based on certain factorizations of the extremal extensions via an auxiliary Hilbert space $\mathfrak{H}_{S}$ constructed from the initial, not necessarily closable, nonnegative operator (or relation) $S$. Then suitable closable operators $R: \mathfrak{H} \rightarrow \mathfrak{H}_{S}$ are defined such that $R^{*} R^{* *}$ extends $S$ (see Theorem 2.3 below; cf. [9]). Since the methods needed here do not become essentially more involved, the most general situation is taken up by allowing the original $A$ and $B$ to be multi-valued. Moreover, the so-called form sum that can always be constructed for $A+B$ via the nonnegative quadratic forms

Received by the editors March 27, 2006 and, in revised form, June 15, 2006.

2000 Mathematics Subject Classification. Primary 47A57, 47B25; Secondary 47A55, 47B65.

Key words and phrases. Nonnegative selfadjoint relation, Friedrichs extension, Kreı̆n-von Neumann extension, extremal extension, form sum extension.

The fourth author was supported by the "Fond zur Förderung der wissenschaftlichen Forschung" (FWF, Austria), grant number P15540-N05. 
associated with $A$ and $B$ will be analyzed as a nonnegative selfadjoint (relation) extension of $A+B$.

To outline some of the results more precisely consider the auxiliary Hilbert space $\mathfrak{H}_{A+B}$, which is obtained by providing the quotient space

$$
\operatorname{ran}(A+B) / \operatorname{mul}(A+B)
$$

with a suitable inner product and then completing it. Applying the recent results in 9] all extremal extensions of $A+B$ can then be factorized via $\mathfrak{H}_{A+B}$ in the form $R^{*} R^{* *}$ with some closable operator $R: \mathfrak{H} \rightarrow \mathfrak{H}_{A+B}$. In particular, the Krel̆n-von Neumann extension $(A+B)_{N}$ and the Friedrichs extension $(A+B)_{F}$ of $A+B$ can be factorized in this way. Here $R$ need not be densely defined. Necessarily $\operatorname{dom} R \subset \operatorname{dom}(A+B)_{N}^{\frac{1}{2}}$, but also $\operatorname{dom}(A+B)_{N}^{\frac{1}{2}}$ need not be dense in $\mathfrak{H}$, which reflects the fact that $A+B$ need not be positively closable. It is shown that the space $\mathfrak{H}_{A+B}$ can be identified as a closed subspace $\mathfrak{E}$ of the Cartesian product space $\mathfrak{H} \times \mathfrak{H}$. This leads to more explicit formulas for the class of extremal extensions; all of them are factorized via $\mathfrak{H} \times \mathfrak{H}$ with closable operators $T: \mathfrak{H} \rightarrow \mathfrak{H} \times \mathfrak{H}$ with $\operatorname{ran} T \subset \mathfrak{E}$ (see Theorems 3.3, 3.4). The explicit factorizations constructed via the space $\mathfrak{H} \times \mathfrak{H}$ make it natural to study the above mentioned form sum extension and its relation to extremal extensions of $A+B$. It is shown that the form sum extension need not be extremal (see Example 5.2); a criterion for its extremality is established in Theorem 4.1, indicating a nontrivial dependence on $A$ and $B$.

The factorization of nonnegative selfadjoint extensions of a nonnegative operator $S$ over an auxiliary Hilbert space related to $S$ is a procedure which goes back to Z. Sebestyén and J. Stochel [10, 11]. It was further developed in [1], 9]. The factorization of the form sum extension in the case that $A, B$, and $A+B$ are densely defined operators goes back to B. Farkas and M. Matolcsi [4, [5]; in the case that $A+B$ is a nondensely defined operator, see $[8]$.

\section{Preliminary Results}

2.1. Some terminology. A linear relation from a Hilbert space $\mathfrak{H}$ to a Hilbert space $\mathfrak{K}$ is a linear subspace of the Cartesian product $\mathfrak{H} \times \mathfrak{K}$. The following selfexplanatory notions domain, range, kernel, and multi-valued part of $A$ will be used throughout the paper:

$$
\begin{aligned}
\operatorname{dom} A & =\left\{f \in \mathfrak{H}:\left\{f, f^{\prime}\right\} \in A\right\}, \quad \operatorname{ran} A=\left\{f^{\prime} \in \mathfrak{K}:\left\{f, f^{\prime}\right\} \in A\right\}, \\
\operatorname{ker} A & =\{f \in \mathfrak{H}:\{f, 0\} \in A\}, \quad \operatorname{mul} A=\left\{f^{\prime} \in \mathfrak{K}:\left\{0, f^{\prime}\right\} \in A\right\} .
\end{aligned}
$$

The closures of $\operatorname{dom} A$ and $\operatorname{ran} A$ in $\mathfrak{H}$ and $\mathfrak{K}$, respectively, will be denoted by $\overline{\operatorname{dom}} A$ and $\overline{\operatorname{ran}} A$. The formal inverse $A^{-1}$ is defined as $A^{-1}=\left\{\left\{f^{\prime}, f\right\}:\left\{f, f^{\prime}\right\} \in A\right\}$; it is a linear relation from $\mathfrak{K}$ to $\mathfrak{H}$. Observe the following formal identities $\operatorname{dom} A^{-1}=$ $\operatorname{ran} A$ and $\operatorname{ker} A^{-1}=\operatorname{mul} A$. The relation $A$ is closed if it is closed as a subspace of $\mathfrak{H} \times \mathfrak{K}$; the closure of the relation $A$ is the closure of the subspace $A$ in $\mathfrak{H} \times \mathfrak{K}$. If $A$ is closed, then the subspaces $\operatorname{ker} A$ and mul $A$ are closed. A linear relation $A$ is the graph of an operator if and only if mul $A=\{0\}$. In the present context a linear operator $A$ from $\mathfrak{H}$ to $\mathfrak{K}$ is identitfied with its graph. It is said to be closable if its closure is the graph of an operator.

The adjoint of a linear relation $A$ from $\mathfrak{H}$ to $\mathfrak{K}$ is the closed linear relation $A^{*}$ from $\mathfrak{K}$ to $\mathfrak{H}$ defined by

$$
A^{*}=\left\{\left\{f, f^{\prime}\right\} \in \mathfrak{K} \times \mathfrak{H}:\left(f^{\prime}, h\right)=\left(f, h^{\prime}\right) \text { for all }\left\{h, h^{\prime}\right\} \in A\right\} .
$$


Observe that $\left(A^{-1}\right)^{*}=\left(A^{*}\right)^{-1}$, so that $(\operatorname{dom} A)^{\perp}=\operatorname{mul} A^{*}$ and $(\operatorname{ran} A)^{\perp}=\operatorname{ker} A^{*}$. Clearly the double adjoint $A^{* *}$ is the closure of the relation $A$. A linear relation $A$ in a Hilbert space $\mathfrak{H}$ (i.e., from $\mathfrak{H}$ to $\mathfrak{H}$ ) is said to be symmetric if $A \subset A^{*}$, or equivalently, if $\left(f^{\prime}, f\right) \in \mathbb{R}$ for all $\left\{f, f^{\prime}\right\} \in A$. A linear relation $A$ in a Hilbert space $\mathfrak{H}$ is said to be nonnegative if $\left(f^{\prime}, f\right) \geq 0$ for all $\left\{f, f^{\prime}\right\} \in A$. A linear relation $A$ in a Hilbert space $\mathfrak{H}$ is said to be selfadjoint if $A^{*}=A$ (so that it is automatically closed); it is said to be essentially selfadjoint if its closure is equal to $A^{*}$. For any closed linear relation $A$ the relation $A_{\infty}=\{0\} \times \operatorname{mul} A$ is selfadjoint in the Hilbert space mul $A$. A selfadjoint relation can always be orthogonally decomposed as $A=A_{s} \oplus A_{\infty}$, where $A_{s}$ is a (densely defined) selfadjoint operator in $\mathfrak{H} \ominus \operatorname{mul} A=\overline{\operatorname{dom}} A$.

2.2. Some block representations with unbounded and multi-valued entries. Let $\mathfrak{H}, \mathfrak{K}, \mathfrak{H}_{i}$, and $\mathfrak{K}_{i}, i=1,2$, be Hilbert spaces. Let $A$ be a linear relation from $\mathfrak{H}$ to $\mathfrak{K}_{1}$, let $B$ be a linear relation from $\mathfrak{H}$ into $\mathfrak{K}_{2}$, and define a linear relation from $\mathfrak{H}$ into $\mathfrak{K}_{1} \times \mathfrak{K}_{2}$ by

$$
\operatorname{col}(A, B):=\left(\begin{array}{l}
A \\
B
\end{array}\right)=\left\{\left\{h, k_{1} \oplus k_{2}\right\}:\left\{h, k_{1}\right\} \in A,\left\{h, k_{2}\right\} \in B\right\} .
$$

Then the following relations hold:

$$
\begin{aligned}
& \operatorname{dom} \operatorname{col}(A, B)=\operatorname{dom} A \cap \operatorname{dom} B, \\
& \operatorname{ker} \operatorname{col}(A, B)=\operatorname{ker} A \cap \operatorname{ker} B, \\
& \operatorname{ran} \operatorname{col}(A, B)=\{A(h) \times B(h): h \in \operatorname{dom} A \cap \operatorname{dom} B\}, \\
& \operatorname{mul} \operatorname{col}(A, B)=\operatorname{mul} A \times \operatorname{mul} B .
\end{aligned}
$$

Here the image of $\mathfrak{M}$ under $A: \mathfrak{H} \rightarrow \mathfrak{K}$ is denoted by $A(\mathfrak{M})=\{k \in \mathfrak{H}:\{h, k\} \in$ $A$ for some $h \in \mathfrak{M}\}$. Moreover, let $C$ be a linear relation from $\mathfrak{H}_{1}$ to $\mathfrak{K}$, let $D$ be a linear relation from $\mathfrak{H}_{2}$ into $\mathfrak{K}$, and define a linear relation from $\mathfrak{H}_{1} \times \mathfrak{H}_{2}$ to $\mathfrak{K}$ by

$$
(C \quad D)=\left\{\left\{h_{1} \oplus h_{2}, k_{1}+k_{2}\right\}:\left\{h_{1}, k_{1}\right\} \in C, \quad\left\{h_{2}, k_{2}\right\} \in D\right\} .
$$

Now the following relations hold:

$$
\begin{aligned}
& \operatorname{dom}\left(\begin{array}{ll}
C & D
\end{array}\right)=\operatorname{dom} C \times \operatorname{dom} D, \\
& \operatorname{ker}\left(\begin{array}{ll}
C & D
\end{array}\right)=\left\{C^{-1}(k) \times D^{-1}(-k): k \in \operatorname{ran} C \cap \operatorname{ran} D\right\}, \\
& \operatorname{ran}(C \quad D)=\operatorname{ran} C+\operatorname{ran} D, \\
& \operatorname{mul}\left(\begin{array}{ll}
C & D
\end{array}\right)=\operatorname{mul} C+\operatorname{mul} D .
\end{aligned}
$$

Here $A^{-1}(\mathfrak{L})$ stands for the preimage of $\mathfrak{L}$ under $A: \mathfrak{H} \rightarrow \mathfrak{K}$ :

$$
A^{-1}(\mathfrak{L})=\{h \in \mathfrak{H}:\{h, k\} \in A \text { for some } k \in \mathfrak{L}\} .
$$

Proposition 2.1. Let $A, B, C$, and $D$ be relations as above. Then:

(i) the adjoint of the linear relation in (2.1) satisfies

$$
\left(\begin{array}{l}
A \\
B
\end{array}\right)^{*} \supset\left(\begin{array}{ll}
A^{*} & B^{*}
\end{array}\right)
$$

(ii) the adjoint of the linear relation in (2.2) satisfies

$$
\left(\begin{array}{ll}
C & D
\end{array}\right)^{*}=\left(\begin{array}{l}
C^{*} \\
D^{*}
\end{array}\right) ;
$$

(iii) if $A$ (or $B$ ) is bounded and densely defined with $\operatorname{dom} B \subset \operatorname{dom} A$ (with $\operatorname{dom} A \subset \operatorname{dom} B$, respectively), then equality holds in (i). 
Proof. (i) Let $\left\{f_{1}, g_{1}\right\} \in A^{*}$ and $\left\{f_{2}, g_{2}\right\} \in B^{*}$. Then

$$
\left(g_{1}+g_{2}, h\right)-\left(f_{1} \oplus f_{2}, k_{1} \oplus k_{2}\right)=\left(g_{1}, h\right)-\left(f_{1}, k_{1}\right)+\left(g_{2}, h\right)-\left(f_{2}, k_{2}\right)=0
$$

holds for all $\left\{h, k_{1}\right\} \in A$ and $\left\{h, k_{2}\right\} \in B$.

(ii) Let $\left\{f, g_{1}\right\} \in C^{*}$ and $\left\{f, g_{2}\right\} \in D^{*}$. Then

$$
\left(g_{1} \oplus g_{2}, h_{1} \oplus h_{2}\right)-\left(f, k_{1}+k_{2}\right)=\left(g_{1}, h_{1}\right)-\left(f, k_{1}\right)+\left(g_{2}, h_{2}\right)-\left(f, k_{2}\right)=0
$$

holds for all $\left\{h_{1}, k_{1}\right\} \in C$ and $\left\{h_{2}, k_{2}\right\} \in D$, which proves the inclusion " $\supset$ ".

Now assume that $\left\{f, g_{1} \oplus g_{2}\right\} \in \mathfrak{K} \times\left(\mathfrak{H}_{1} \oplus \mathfrak{H}_{2}\right)$ belongs to $(C D)^{*}$, so that

$$
\left(g_{1} \oplus g_{2}, h_{1} \oplus h_{2}\right)-\left(f, k_{1}+k_{2}\right)=0
$$

is satisfied for all $\left\{h_{1}, k_{1}\right\} \in C$ and $\left\{h_{2}, k_{2}\right\} \in D$. By taking $h_{1}=k_{1}=0$ it follows from (2.3) that $\left\{f, g_{2}\right\} \in D^{*}$ and similarly by taking $h_{2}=k_{2}=0$ one obtains $\left\{f, g_{1}\right\} \in C^{*}$. This proves the reverse inclusion " $\subset$ ".

(iii) Assume that for instance $B$ is bounded and densely defined. Then $B^{*}$ is bounded and everywhere defined, and $\left\{f_{1} \oplus f_{2}, g\right\} \in\left(\mathfrak{K}_{1} \times \mathfrak{K}_{2}\right) \times \mathfrak{H}$ belongs to $(\operatorname{col}(A, B))^{*}$ if and only if

$0=(g, h)-\left(f_{1} \oplus f_{2}, k_{1} \oplus k_{2}\right)=(g, h)-\left(f_{1}, k_{1}\right)-\left(B^{*} f_{2}, h\right)=\left(g-B^{*} f_{2}, h\right)-\left(f_{1}, k_{1}\right)$ holds for all $\left\{h, k_{1}\right\} \in A$ and $\left\{h, k_{2}\right\} \in B$. Here $\operatorname{dom} A \cap \operatorname{dom} B=\operatorname{dom} A$ and therefore $\left\{f_{1}, g-B^{*} f_{2}\right\} \in A^{*}$. This proves the reverse inclusion " $\subset$ " in (i).

The restriction of a relation $A: \mathfrak{H} \rightarrow \mathfrak{K}$ to the subspace $\mathfrak{M} \subset \mathfrak{H}$ is defined by

$$
A\lceil\mathfrak{M}=\{\{h, k\} \in A: h \in \mathfrak{M}\} .
$$

The adjoint of the restriction of $A$ to $\mathfrak{M}$ satisfies $\left(A\lceil\mathfrak{M})^{*} \supset P_{\mathfrak{M}} A^{*}\right.$, where $P_{\mathfrak{M}}$ is the orthogonal projection onto the closure clos $\mathfrak{M}$ of $\mathfrak{M}$. In particular,

$$
\text { if } \operatorname{dom} A \subset \mathfrak{M} \text {, then }\left(A\lceil\mathfrak{M})^{*}=P_{\mathfrak{M}} A^{*}, \quad\left(A\lceil\mathfrak{M})^{* *}=A^{* *}\lceil\operatorname{clos} \mathfrak{M} .\right.\right.
$$

Here the identity for the adjoints is also a special case of (ii) in Proposition 2.1 with $D=\{0,0\}$. If $P_{\mathfrak{L}}$ is an orthogonal projection onto a closed subspace $\mathfrak{L} \subset \mathfrak{K}$, then $\left(P_{\mathfrak{L}} A\right)^{*}=A^{*} P_{\mathfrak{L}}$ holds for any linear relation $A$. In particular, if $\operatorname{ran} A \subset \mathfrak{L}$ or equivalently $A=P_{\mathfrak{L}} A$, then $A^{*}=A^{*} P_{\mathfrak{L}}$ and $A^{* *}=P_{\mathfrak{L}} A^{* *}$. In this case $A$ can be viewed as a relation $A_{r}$ from $\mathfrak{H}$ to $\mathfrak{L}$, in which case

$$
A_{r}^{*}=A^{*} \uparrow \mathfrak{L}, \quad A_{r}^{* *}=P_{\mathfrak{L}} A^{* *} .
$$

Here the identity for $A_{r}^{*}$ is also obtained from part (iii) in Proposition 2.1 with $B=0$ (i.e., $B f=0$ for all $f \in \mathfrak{H}$ ). These simple observations will be useful in the construction of the factorizations for nonnegative selfadjoint relations in view of

$$
\left(A \lceil \mathfrak { M } ) ^ { * * } \left(A\lceil\mathfrak{M})^{*}=A^{* *} A^{*}, \quad A_{r}^{*} A_{r}^{* *}=A^{*} A^{* *} .\right.\right.
$$

2.3. A factorization of extremal extensions. Let $S$ be a nonnegative linear relation in a Hilbert space $\mathfrak{H}$. Provide the linear space $\operatorname{ran} S$ with the semi-inner product $\langle\cdot, \cdot\rangle$ defined by

$$
\left\langle f^{\prime}, g^{\prime}\right\rangle:=\left(f^{\prime}, g\right)=\left(f, g^{\prime}\right), \quad\left\{f, f^{\prime}\right\},\left\{g, g^{\prime}\right\} \in S .
$$

Define the linear space $\mathfrak{R}_{0} \subset \operatorname{ran} S$ by

$$
\mathfrak{R}_{0}=\left\{f^{\prime}:\left(f^{\prime}, f\right)=0 \text { for some }\left\{f, f^{\prime}\right\} \in S\right\} .
$$


Then $\mathfrak{R}_{0}=\operatorname{ran} S \cap \operatorname{mul} S^{*}$; cf. [9, Lemma 4.1]. Clearly, the quotient space $\operatorname{ran} S / \mathfrak{R}_{0}$ is a pre-Hilbert space with the inner product

$$
\left\langle\left[f^{\prime}\right],\left[g^{\prime}\right]\right\rangle:=\left(f^{\prime}, g\right)=\left(f, g^{\prime}\right), \quad\left\{f, f^{\prime}\right\},\left\{g, g^{\prime}\right\} \in S,
$$

where $\left[f^{\prime}\right],\left[g^{\prime}\right]$ denote the equivalence classes containing $f^{\prime}$ and $g^{\prime}$. Let $\mathfrak{H}_{S}$ be the Hilbert space completion of $\operatorname{ran} S / \mathfrak{R}_{0}$, whose inner product is again denoted by $\langle\cdot, \cdot\rangle$. The linear relation $Q$ from $\mathfrak{H}$ to $\mathfrak{H}_{S}$ is defined by

$$
Q=\left\{\left\{f,\left[f^{\prime}\right]\right\}:\left\{f, f^{\prime}\right\} \in S\right\},
$$

so that actually $Q$ is (the graph of) an operator. Define the linear relation $J$ from $\mathfrak{H}_{S}$ to $\mathfrak{H}$ by

$$
\left.J=\left\{\left\{f^{\prime}\right], f^{\prime}\right\}:\left\{f, f^{\prime}\right\} \in S\right\},
$$

so that mul $J=\mathfrak{R}_{0}$. Since $J$ is densely defined in $\mathfrak{H}_{S}, J^{*}$ is an operator. The definitions (2.9) and (2.10) imply that $J \subset Q^{*}$ and $Q \subset J^{*}$. In particular $Q^{* *}$, the closure of $Q$, is an operator. Now one can state the following factorization result established in the general case in [9, Theorem 4.3]; in the case that $S$ is densely defined, see [1, Proposition 3.1].

Theorem $2.2([1,9])$. Let $S$ be a nonnegative relation in a Hilbert space $\mathfrak{H}$ and let $J$ and $Q$ be defined by (2.9) and (2.10). Then the Krĕn-von Neumann extension $S_{N}$ of $S$ is given by $S_{N}=J^{* *} J^{*}$ and the corresponding closed form $\mathfrak{t}_{N}$ is given by

$$
\mathfrak{t}_{N}[f, g]=\left\langle J^{*} f, J^{*} g\right\rangle, \quad f, g \in \operatorname{dom} J^{*}=\operatorname{dom} S_{N}^{\frac{1}{2}} .
$$

Furthermore, the Friedrichs extension $S_{F}$ of $S$ is given by $S_{F}=Q^{*} Q^{* *}$ and the corresponding closed form $\mathfrak{t}_{F}$ is given by

$$
\mathfrak{t}_{F}[f, g]=\left\langle Q^{* *} f, Q^{* *} g\right\rangle, \quad f, g \in \operatorname{dom} Q^{* *}=\operatorname{dom} S_{F}^{\frac{1}{2}} .
$$

A nonnegative selfadjoint extension $\widetilde{A}$ of $S$ is called extremal when

$$
\inf \left\{\left(f^{\prime}-h^{\prime}, f-h\right):\left\{h, h^{\prime}\right\} \in S\right\}=0 \quad \text { for all } \quad\left\{f, f^{\prime}\right\} \in \widetilde{A}
$$

see [2]. The Kreŭn-von Neumann extension $S_{N}$ and the Friedrichs extension $S_{F}$ are extremal extensions. Let $\mathfrak{L}$ be any subspace such that

$$
\operatorname{dom} S \subset \mathfrak{L} \subset \operatorname{dom} J^{*}=\operatorname{dom} S_{N}^{\frac{1}{2}},
$$

and associate with $\mathfrak{L}$ the restriction operator $R_{\mathfrak{L}}$ from $\mathfrak{H}_{\text {to }} \mathfrak{H}_{S}$ by

$$
R_{\mathfrak{L}}:=J^{*} \uparrow \mathfrak{L}=\left\{\left\{f, f^{\prime}\right\} \in J^{*}: f \in \mathfrak{L}\right\} .
$$

Since $J^{*}$ is a closed operator from $\mathfrak{H}$ to $\mathfrak{H}_{S}$, it is clear that $R_{\mathfrak{L}}$ is a closable operator. The definition of $R_{\mathfrak{L}}$ and Theorem 2.2 lead to

$$
\left\langle R_{\mathfrak{L}} f, R_{\mathfrak{L}} g\right\rangle=\left\langle J^{*} f, J^{*} g\right\rangle=\mathfrak{t}_{N}[f, g], \quad f, g \in \mathfrak{L} .
$$

Hence, $R_{\mathfrak{L}}$ is closed if and only if the restriction to $\mathfrak{L}$ of the form $\mathfrak{t}_{N}[\cdot, \cdot]$ is closed. Clearly, operators of the form $R_{\mathfrak{L}}$ induce nonnegative selfadjoint relations $R_{\mathfrak{L}}^{*} R_{\mathfrak{L}}^{* *}$, and the corresponding closed nonnegative forms $\mathfrak{t}_{\mathfrak{L}}$ are given by

$$
\mathfrak{t}_{\mathfrak{L}}[f, g]=\left\langle R_{\mathfrak{L}}^{* *} f, R_{\mathfrak{L}}^{* *} g\right\rangle \quad f, g \in \operatorname{dom} R_{\mathfrak{L}}^{* *} .
$$

This yields the following useful characterization of extremal extensions of $S$; again the general case is established in [9. Theorem 6.1] and the densely defined case in [1, Proposition 4.1]. For another approach see [7. 
Theorem 2.3 ([1, 9]). Let $S$ be a nonnegative relation in a Hilbert space $\mathfrak{H}$. Then the following statements are equivalent:

(i) $\widetilde{A}$ is an extremal extension of $S$;

(ii) $\widetilde{A}=R_{\mathfrak{L}}^{*} R_{\mathfrak{L}}^{* *}$ for some subspace $\mathfrak{L}$ such that $\operatorname{dom} S \subset \mathfrak{L} \subset \operatorname{dom} S_{N}^{\frac{1}{2}}$;

(iii) $\widetilde{A}$ is a nonnegative selfadjoint extension of $S$ whose corresponding form $\tilde{\mathfrak{t}}$ satisfies $\tilde{\mathfrak{t}} \subset \mathfrak{t}_{N}$.

As an example consider the purely multi-valued relation $S$ in $\mathfrak{H}$ defined by

$$
S=\{0\} \times \mathfrak{K},
$$

where $\mathfrak{K}$ is a not necessarily closed subspace of the Hilbert space $\mathfrak{H}$. Then $S$ is closed if and only if $\mathfrak{K}$ is closed. The adjoint $S^{*}$ is given by

$$
S^{*}=\mathfrak{K}^{\perp} \times \mathfrak{H},
$$

so that mul $S^{*}=\mathfrak{H}$. Since $S$ is nonnegative, the above construction is applicable and $\mathfrak{R}_{0}=\operatorname{ran} S=\mathfrak{K}, \mathfrak{H}_{S}=\{[0]\}, Q=\{\{0,[0]\}\}$, and $J=\{[0]\} \times \mathfrak{K}$, so that $Q^{*}=\{[0]\} \times \mathfrak{H}, Q^{* *}=\{0\} \times\{[0]\}, J^{*}=\mathfrak{K}^{\perp} \times\{[0]\}$, and $J^{* *}=\{[0]\} \times \overline{\mathfrak{K}}$. Therefore, according to Theorem 2.2, the Kreı̆n-von Neumann extension $S_{N}=J^{* *} J^{*}$ and the Friedrichs extension $S_{F}=Q^{*} Q^{* *}$ are given by

$$
S_{N}=\mathfrak{K}^{\perp} \times \overline{\mathfrak{K}}, \quad S_{F}=\{0\} \times \mathfrak{H} ;
$$

cf. [3. The form corresponding to $S_{N}$ is the null form defined on all of $\mathfrak{K}^{\perp}$, and the form corresponding to $S_{F}$ is the null form on $\{0\}$. This gives the following result.

Corollary 2.4. Let the nonnegative relation $S$ be given by (2.13). Then there exists a one to-one-correspondence between the class of all extremal extensions $\widetilde{A}$ of $S$ and the set of all closed subspaces $\mathfrak{L}$ of $\mathfrak{K}^{\perp}$. The correspondence is given by

$$
\widetilde{A}=\mathfrak{L} \times \mathfrak{L}^{\perp},
$$

and the associated form $\tilde{\mathfrak{t}}$ is the null form on $\mathfrak{L}$.

\section{Construction of extremal extensions for the sum}

3.1. Pairs of nonnegative selfadjoint relations. Let $A$ and $B$ be nonnegative selfadjoint relations in a Hilbert space $\mathfrak{H}$. Then the sum $A+B$ is the relation in $\mathfrak{H}$ defined by (1.1). Note that

$$
\operatorname{mul}(A+B)=\operatorname{mul} A+\operatorname{mul} B .
$$

If $A$ and $B$ are decomposed as $A=A_{s} \oplus A_{\infty}$ and $B=B_{s} \oplus B_{\infty}$, where $A_{\infty}=$ $\{0\} \times \operatorname{mul} A, B_{\infty}=\{0\} \times \operatorname{mul} B$, and $A_{s}$ and $B_{s}$ are densely defined nonnegative selfadjoint operators (defined as orthogonal complements in the graph sense), then the uniquely determined square roots of $A$ and $B$ are given by

$$
A^{\frac{1}{2}}=A_{s}^{\frac{1}{2}} \oplus A_{\infty}, \quad B^{\frac{1}{2}}=B_{s}^{\frac{1}{2}} \oplus B_{\infty} .
$$

Associate with $A$ and $B$ the relation $\Phi$ from $\mathfrak{H} \times \mathfrak{H}$ to $\mathfrak{H}$, defined by

$$
\Phi=\left\{\left\{f \oplus g, f^{\prime}+g^{\prime}\right\}:\left\{f, f^{\prime}\right\} \in A^{\frac{1}{2}},\left\{g, g^{\prime}\right\} \in B^{\frac{1}{2}}\right\} .
$$

Clearly, the domain and the multi-valued part of $\Phi$ are given by

$$
\operatorname{dom} \Phi=\operatorname{dom} A^{\frac{1}{2}} \times \operatorname{dom} B^{\frac{1}{2}}, \quad \operatorname{mul} \Phi=\operatorname{mul} A+\operatorname{mul} B .
$$


Lemma 3.1. The adjoint $\Phi^{*}$ of $\Phi$ is the relation from $\mathfrak{H}$ to $\mathfrak{H} \times \mathfrak{H}$, given by

$$
\Phi^{*}=\left\{\{h, k \oplus l\}:\{h, k\} \in A^{\frac{1}{2}},\{h, l\} \in B^{\frac{1}{2}}\right\} .
$$

Proof. Since $\Phi=\left(\begin{array}{ll}A^{\frac{1}{2}} & B^{\frac{1}{2}}\end{array}\right)$, (3.4) is obtained from part (ii) in Proposition 2.1.

Clearly, $\operatorname{dom} \Phi^{*}=\operatorname{dom} A^{\frac{1}{2}} \cap \operatorname{dom} B^{\frac{1}{2}}, \operatorname{mul} \Phi^{*}=\operatorname{mul} A \times \operatorname{mul} B$, and one can define the (orthogonal) operator part $\left(\Phi^{*}\right)_{s}$ of $\Phi^{*}$ by

$$
\left(\Phi^{*}\right)_{s}=\left(\begin{array}{c}
A_{s}^{\frac{1}{2}} \\
B_{s}^{\frac{1}{2}}
\end{array}\right)=\left\{\left\{h, A_{s}^{\frac{1}{2}} h \oplus B_{s}^{\frac{1}{2}} h\right\}: h \in \operatorname{dom} A^{\frac{1}{2}} \cap \operatorname{dom} B^{\frac{1}{2}}\right\} .
$$

Now define the relation $\Psi$ from $\mathfrak{H}$ to $\mathfrak{H} \times \mathfrak{H}$ by

$$
\Psi=\left\{\left\{h, A_{s}^{\frac{1}{2}} h \oplus B_{s}^{\frac{1}{2}} h\right\}: h \in \operatorname{dom} A \cap \operatorname{dom} B\right\} \subset \mathfrak{H} \times(\mathfrak{H} \times \mathfrak{H}) .
$$

Then $\Psi$ is an operator with $\operatorname{dom} \Psi=\operatorname{dom} A \cap \operatorname{dom} B$. In fact $\Psi \subset\left(\Phi^{*}\right)_{s}$, so that $\Psi$ is a closable operator. Denote by $\mathfrak{F}_{0}$ the range of $\left(\Phi^{*}\right)_{s}$ and let $\mathfrak{E}_{0}$ be the range of $\Psi$ and let their closures in $\mathfrak{H} \times \mathfrak{H}$ be denoted by $\mathfrak{F}$ and $\mathfrak{E}$, respectively. Then

$$
\mathfrak{E}_{0}=\operatorname{ran} \Psi \subset \operatorname{ran}\left(\Phi^{*}\right)_{s}=\mathfrak{F}_{0} \text { and } \mathfrak{E} \subset \mathfrak{F} .
$$

It follows from $\overline{\operatorname{dom}} \Psi^{*}=\left(\operatorname{mul} \Psi^{* *}\right)^{\perp}$ and $\operatorname{mul} \Psi^{*}=(\operatorname{dom} \Psi)^{\perp}$ that

$$
\overline{\operatorname{dom}} \Psi^{*}=\mathfrak{H}, \quad \operatorname{mul} \Psi^{*}=(\operatorname{dom} A \cap \operatorname{dom} B)^{\perp} .
$$

Next define the linear relation $K$ from $\mathfrak{H} \times \mathfrak{H}$ to $\mathfrak{H}$ by

$$
K=\left\{\left\{A_{s}^{\frac{1}{2}} f \oplus B_{s}^{\frac{1}{2}} f, f^{\prime}+f^{\prime \prime}\right\}:\left\{A_{s}^{\frac{1}{2}} f, f^{\prime}\right\} \in A^{\frac{1}{2}},\left\{B_{s}^{\frac{1}{2}} f, f^{\prime \prime}\right\} \in B^{\frac{1}{2}}\right\} .
$$

Clearly, the domain and the multi-valued part of $K$ are given by

$$
\operatorname{dom} K=\mathfrak{E}_{0}, \quad \operatorname{mul} K=\operatorname{mul}(A+B) .
$$

Lemma 3.2. The relations $K, \Phi$, and $\Psi$ satisfy the following inclusions:

$$
K \subset \Phi \subset \Psi^{*}, \quad \Psi \subset \Phi^{*} \subset K^{*} .
$$

Proof. To see this note that $K \subset \Phi$ follows from (3.2) and (3.8), and that $\Psi \subset \Phi^{*}$ follows from (3.4) and (3.6). Therefore, also $\Phi^{*} \subset K^{*}$ and $\Phi \subset \Phi^{* *} \subset \Psi^{*}$.

3.2. Factorization of extremal extensions via $\mathfrak{H} \times \mathfrak{H}$. Let $A$ and $B$ be nonnegative selfadjoint relations and let $\mathfrak{R}_{0}$ be defined by (2.7) with $S=A+B$. Then $\mathfrak{R}_{0}=\operatorname{mul}(A+B)$, and the corresponding quotient space is

$$
\operatorname{ran} S / \mathfrak{R}_{0}=\operatorname{ran}(A+B) / \operatorname{mul}(A+B),
$$

with the inner product given by (2.6) with $S=A+B$. Denote by $\mathfrak{H}_{A+B}$ the completion of the space in (3.10) with respect to the norm $\|\cdot\|_{A+B}$ induced by the inner product (2.6) with $S=A+B$. Define the linear relation $Z_{0}$ by

$$
Z_{0}=\left\{\left\{A_{s}^{\frac{1}{2}} h \oplus B_{s}^{\frac{1}{2}} h,\left[h^{\prime}+h^{\prime \prime}\right]\right\}:\left\{h, h^{\prime}\right\} \in A,\left\{h, h^{\prime \prime}\right\} \in B\right\} .
$$

Then

$$
\left\|A_{s}^{\frac{1}{2}} h \oplus B_{s}^{\frac{1}{2}} h\right\|=\left\|\left[h^{\prime}+h^{\prime \prime}\right]\right\|_{A+B}, \quad\left\{h, h^{\prime}\right\} \in A,\left\{h, h^{\prime \prime}\right\} \in B,
$$

so that the relation $Z_{0}$ is isometric from $\mathfrak{E}_{0}$ onto $\operatorname{ran}(A+B) / \mathrm{mul}(A+B)$; and, in fact, $Z_{0}$ is an operator. Hence the closure $Z$ of $Z_{0}$ is a closed isometric operator from the Hilbert space $\mathfrak{E}$, the closure of $\mathfrak{E}_{0}$, onto the Hilbert space $\mathfrak{H}_{A+B}$. 
Recall that $\Psi$ in $(3.6)$ acts from $\mathfrak{H}$ to $\mathfrak{H} \times \mathfrak{H}$ with $\operatorname{ran} \Psi \subset \mathfrak{E}=\overline{\operatorname{ran}} \Psi$. Hence $\Psi$ can be viewed as an operator $\Psi_{e}$ from $\mathfrak{H}$ to $\mathfrak{E}$, so that $\Psi_{e} \subset \Psi$ are the same sets, interpreted in different spaces. Since $Z$ is an isometry from $\mathfrak{E}$ to $\mathfrak{H}_{A+B}$, the product $Z \Psi_{e}$ is well-defined and

$$
Z \Psi_{e}=\left\{\left\{h, Z\left(A_{s}^{\frac{1}{2}} h \oplus B_{s}^{\frac{1}{2}} h\right)\right\}: h \in \operatorname{dom} A \cap \operatorname{dom} B\right\} .
$$

Since $Z\left(A_{s}^{\frac{1}{2}} h \oplus B_{s}^{\frac{1}{2}} h\right)=\left[h^{\prime}+h^{\prime \prime}\right]$ by (3.11), and $\left\{h, h^{\prime}+h^{\prime \prime}\right\} \in A+B$, it follows from (2.9) and (3.12) that the operators $Q$ and $\Psi_{e}$ are connected by

$$
Q=Z \Psi_{e}
$$

Likewise, the relation $K$ in $(3.8)$ is defined as a relation from $\mathfrak{H} \times \mathfrak{H}$ to $\mathfrak{H}$, and $\operatorname{dom} K \subset \mathfrak{E}$; cf. (3.9). Hence $K$ can be viewed as a relation $K_{e}$ from $\mathfrak{E}$ to $\mathfrak{H}$, so that $K_{e} \subset K$ are the same sets, interpreted in different spaces. Since $Z$ is an isometry from $\mathfrak{E}$ to $\mathfrak{H}_{A+B}$, the product $K_{e} Z^{*}$ is well-defined and

$$
K_{e} Z^{*}=\left\{\{\varphi, \psi\}:\{\varphi, \chi\} \in Z^{*},\{\chi, \psi\} \in K_{e} \text { for some } \chi \in \mathfrak{E}\right\} ;
$$

note that $\{\varphi, \chi\} \in Z^{*}$ if and only if $\varphi=Z \chi$. In view of (3.8) this leads to

$$
K_{e} Z^{*}=\left\{\left\{Z\left(A_{s}^{\frac{1}{2}} h \oplus B_{s}^{\frac{1}{2}} h\right), h^{\prime}+h^{\prime \prime}\right\}:\left\{A_{s}^{\frac{1}{2}} h, h^{\prime}\right\} \in A^{\frac{1}{2}},\left\{B_{s}^{\frac{1}{2}} h, h^{\prime \prime}\right\} \in B^{\frac{1}{2}}\right\} .
$$

This means that the linear relations $J$ in $(2.10)$ and $K_{e}$ are connected by

$$
J=K_{e} Z^{*} \text {. }
$$

With these identifications of $Q$ and $J$ in terms of $\Psi_{e}$ and $K_{e}$, the Kre $\breve{n-v o n ~ N e u-~}$ mann and the Friedrichs extensions of $A+B$ can now be expressed in terms of $K$ and $\Psi$.

Theorem 3.3. Let $A$ and $B$ be nonnegative selfadjoint relations. Then:

(i) the Krĕn-von Neumann extension $(A+B)_{N}$ is given by $K^{* *} K^{*}$;

(ii) the Friedrichs extension $(A+B)_{F}$ is given by $\Psi^{*} \Psi^{* *}$.

Proof. (i) Since $Z$ is an isometric operator from $\mathfrak{E}$ onto $\mathfrak{H}_{A+B}$, (3.14) implies that

$$
Z\left(K_{e}\right)^{*}=J^{*}, \quad\left(K_{e}\right)^{* *} Z^{*}=J^{* *} .
$$

Multiplication of the relations in 3.15 shows that

$$
\left(K_{e}\right)^{* *}\left(K_{e}\right)^{*}=\left(K_{e}\right)^{* *} Z^{*} Z\left(K_{e}\right)^{*}=J^{* *} J^{*} .
$$

Since $\left(K_{e}\right)^{* *}\left(K_{e}\right)^{*}=K^{* *} K^{*}$, see (2.5), Theorem 2.2 gives $K^{* *} K^{*}=(A+B)_{N}$.

(ii) Now it follows from (3.13) that

$$
\left(\Psi_{e}\right)^{*} Z^{*}=Q^{*}, \quad Z\left(\Psi_{e}\right)^{* *}=Q^{* *},
$$

and multiplication of the relations in (3.17) shows that

$$
\left(\Psi_{e}\right)^{*}\left(\Psi_{e}\right)^{* *}=\left(\Psi_{e}\right)^{*} Z^{*} Z\left(\Psi_{e}\right)^{* *}=Q^{*} Q^{* *} .
$$

By (2.5) $\left(\Psi_{e}\right)^{*}\left(\Psi_{e}\right)^{* *}=\Psi^{*} \Psi^{* *}$ and now Theorem 2.2 gives $\Psi^{*} \Psi^{* *}=(A+B)_{F}$.

Since mul $(A+B)_{N}=\operatorname{mul} K^{* *}$ the relation $A+B$ is positively closable (i.e., the Krĕ̌n-von Neumann extension $(A+B)_{N}$ is an operator; cf. [9, Subsection 5.4]) if and only if the relation $K$ is closable. If either $A$ or $B$ has a positive lower bound, then $A+B$ is positively closable if and only if $A+B$ is closable; cf. 9, Subsection 5.4]. Likewise, $\operatorname{mul}(A+B)_{F}=\operatorname{mul} \Psi^{*}=(\operatorname{dom} A \cap \operatorname{dom} B)^{\perp}$. 
Since $\operatorname{dom} K_{e}=\mathfrak{E}_{0}$ is dense in $\mathfrak{E}, K_{e}^{*}$ is an operator from $\mathfrak{H}$ to $\mathfrak{E}$. For any subspace $\mathfrak{L}$ with $\operatorname{dom} \Psi_{e} \subset \mathfrak{L} \subset \operatorname{dom} K_{e}^{*}$ define the operator $T_{\mathfrak{L} e}$ from $\mathfrak{H}$ to $\mathfrak{E}$ by

$$
T_{\mathfrak{L} e}=K_{e}^{*}\lceil\mathfrak{L} .
$$

By (3.15) this leads to

$$
Z T_{\mathfrak{L} e}=Z K_{e}^{*}\left\lceil\mathfrak{L}=J^{*} \uparrow \mathfrak{L}=R_{\mathfrak{L}}\right.
$$

cf. (2.12), so that $T_{\mathfrak{L} e}=Z^{*} R_{\mathfrak{L}}$ and

$$
T_{\mathfrak{L} e}^{*}=R_{\mathfrak{L}}^{*} Z, \quad T_{\mathfrak{L} e}^{* *}=Z^{*} R_{\mathfrak{L}}^{* *} .
$$

Denote by $T_{\mathfrak{L}}$ the operator $T_{\mathfrak{L} e}$ viewed as an operator from $\mathfrak{H}$ to $\mathfrak{H} \times \mathfrak{H}$. Then (2.5) and (3.19) imply that

$$
T_{\mathfrak{L}}^{*} T_{\mathfrak{L}}^{* *}=T_{\mathfrak{L} e}^{*} T_{\mathfrak{L} e}^{* *}=R_{\mathfrak{L}}^{*} R_{\mathfrak{L}}^{* *} .
$$

Recall that $\operatorname{dom} \Psi_{e}=\operatorname{dom} A \cap \operatorname{dom} B=\operatorname{dom}(A+B)$ and $\operatorname{dom} K_{e}^{*}=\operatorname{dom} J^{*}$. Hence (3.20) and Theorem 2.3 (i), (ii) give the following result.

Theorem 3.4. Let $A$ and $B$ be nonnegative relations in a Hilbert space $\mathfrak{H}$. Then the following statements are equivalent:

(i) $\widetilde{A}$ is a nonnegative selfadjoint extremal extension of $A+B$;

(ii) $\widetilde{A}=T_{\mathfrak{L}}^{*} T_{\mathfrak{L}}^{* *}$ for some $\mathfrak{L}$ such that $\operatorname{dom}(A+B) \subset \mathfrak{L} \subset \operatorname{dom}(A+B)_{N}^{\frac{1}{2}}$.

\section{THE FORM SUM CONSTRUCTION}

The nonnegative selfadjoint relations $A$ and $B$ generate the following closed nonnegative form:

$$
\left(A_{s}^{\frac{1}{2}} h, A_{s}^{\frac{1}{2}} k\right)+\left(B_{s}^{\frac{1}{2}} h, B_{s}^{\frac{1}{2}} k\right), \quad h, k \in \operatorname{dom} A^{\frac{1}{2}} \cap \operatorname{dom} B^{\frac{1}{2}} .
$$

Observe that the restriction of this form to $\operatorname{dom} \Psi^{* *}$ is equal to

$$
\left(\Psi^{* *} h, \Psi^{* *} k\right)=\left(A_{s}^{\frac{1}{2}} h, A_{s}^{\frac{1}{2}} k\right)+\left(B_{s}^{\frac{1}{2}} h, B_{s}^{\frac{1}{2}} k\right), \quad h, k \in \operatorname{dom} \Psi^{* *},
$$

since $\Psi^{* *} \subset\left(\Phi^{*}\right)_{s}$; cf. (3.5). In fact, this shows that the domain $\operatorname{dom} \Psi^{* *}$ is equal to the energy space $\mathfrak{H}[A+B]$ associated with $A+B$; cf. 3]. Thus the form in (4.2) has a natural domain which is in general larger than $\operatorname{dom} \Psi^{* *}$.

Theorem 4.1. Let $A$ and $B$ be nonnegative selfadjoint relations. The nonnegative selfadjoint relation $\Phi^{* *} \Phi^{*}$ is an extension of the nonnegative relation $A+B$, which corresponds to the closed nonnegative form in (4.1). The following statements are equivalent:

(i) $\Phi^{* *} \Phi^{*}$ is an extremal extension of $A+B$;

(ii) $\mathfrak{E}=\mathfrak{F}$, i.e., $\overline{\operatorname{ran}} \Psi=\overline{\operatorname{ran}}\left(\Phi^{*}\right)_{s}$.

Moreover, $\Phi^{* *} P_{\mathfrak{E}}\left(P_{\mathfrak{E}} \Phi^{*}\right)^{* *}=\left(\Phi^{* *} P_{\mathfrak{E}} \Phi^{*}\right)_{F}$ is an extremal extension of $A+B$.

Proof. The form sum (4.1) can be rewritten as

$$
\left(\left(\Phi^{*}\right)_{s} h,\left(\Phi^{*}\right)_{s} k\right), \quad h, k \in \operatorname{dom}\left(\Phi^{*}\right)_{s}=\operatorname{dom} A^{\frac{1}{2}} \cap \operatorname{dom} B^{\frac{1}{2}},
$$

so that $\Phi^{* *} \Phi^{*}$ is the nonnegative selfadjoint relation which corresponds to (4.1); cf. [8, Proposition 5.2]. To show that $\Phi^{* *} \Phi^{*}$ extends $A+B$, let $\left\{h, h_{A}^{\prime}+h_{B}^{\prime}\right\} \in(A+B)$ for some $\left\{h, h_{A}^{\prime}\right\} \in A$ and $\left\{h, h_{B}^{\prime}\right\} \in B$, so that $h \in \operatorname{dom} A \cap \operatorname{dom} B$. Clearly, 
$\left\{h, A_{s}^{\frac{1}{2}} h \oplus B_{s}^{\frac{1}{2}} h\right\} \in \Phi^{*}$. Moreover, $\left\{A_{s}^{\frac{1}{2}} h \oplus B_{s}^{\frac{1}{2}} h, h_{A}^{\prime}+h_{B}^{\prime}\right\} \in \Phi^{* *}$, as can be verified directly:

$$
\left(h_{A}^{\prime}+h_{B}^{\prime}, \varphi\right)-\left(A_{s}^{\frac{1}{2}} h \oplus B_{s}^{\frac{1}{2}} h, A_{s}^{\frac{1}{2}} \varphi \oplus B_{s}^{\frac{1}{2}} \varphi\right)=0, \quad \varphi \in \operatorname{dom} A \cap \operatorname{dom} B .
$$

Therefore $\left\{h, h_{A}^{\prime}+h_{B}^{\prime}\right\} \in \Phi^{* *} \Phi^{*}$. Hence $A+B \subset \Phi^{* *} \Phi^{*}$.

(i) $\Rightarrow$ (ii) In view of (3.7) it is enough to prove that $\mathfrak{F} \subset \mathfrak{E}$. Assume that $\Phi^{* *} \Phi^{*}$ is extremal. Then by Theorem 3.4 there exists a subspace $\mathfrak{L}$ such that

$$
\left(\left(\Phi^{*}\right)_{s}\right)^{*}\left(\Phi^{*}\right)_{s}=\Phi^{* *} \Phi^{*}=T_{\mathfrak{L}}^{*} T_{\mathfrak{L}}^{* *}=T_{\mathfrak{L} e}^{*} T_{\mathfrak{L} e}^{* *} .
$$

By Lemma $3.2\left(\Phi^{*}\right)_{s} \subset \Phi^{*} \subset K^{*}$ and therefore $P_{\mathfrak{E}}\left(\Phi^{*}\right)_{s} \subset P_{\mathfrak{E}} K^{*}=K_{e}^{*}$ with $\operatorname{dom} P_{\mathfrak{E}}\left(\Phi^{*}\right)_{s}=\operatorname{dom}\left(\Phi^{*}\right)_{s}=\operatorname{dom} T_{\mathfrak{L}}^{* *}=\operatorname{dom} T_{\mathfrak{L} e}^{* *} ;$ see (2.4). Since $P_{\mathfrak{E}}\left(\Phi^{*}\right)_{s}$ and $T_{\mathfrak{L} e}^{* *}$ are restrictions of the operator $K_{e}^{*}$ it follows that

$$
P_{\mathfrak{E}}\left(\Phi^{*}\right)_{s}=T_{\mathfrak{L} e}^{* *}, \quad\left(\left(\Phi^{*}\right)_{s}\right)^{*} P_{\mathfrak{E}}=T_{\mathfrak{L} e}^{*} .
$$

Now (4.3) implies that

$$
\left(\left(\Phi^{*}\right)_{s}\right)^{*}\left(\Phi^{*}\right)_{s}=T_{\mathfrak{L} e}^{*} T_{\mathfrak{L} e}^{* *}=\left(\left(\Phi^{*}\right)_{s}\right)^{*} P_{\mathfrak{E}} T_{\mathfrak{L} e}^{* *}=\left(\left(\Phi^{*}\right)_{s}\right)^{*} T_{\mathfrak{L} e}^{* *}
$$

Hence for every $f \in \operatorname{dom}\left(\left(\Phi^{*}\right)_{s}\right)^{*}\left(\Phi^{*}\right)_{s}$ one has $\left(\Phi^{*}\right)_{s} f-T_{\mathcal{L} e}^{* *} f \in \operatorname{ker}\left(\left(\Phi^{*}\right)_{s}\right)^{*}$, and since $\left(\Phi^{*}\right)_{s} f-T_{\mathfrak{L} e}^{* *} f \in \mathfrak{F}=\overline{\operatorname{ran}}\left(\Phi^{*}\right)_{s}=\left(\operatorname{ker}\left(\left(\Phi^{*}\right)_{s}\right)^{*}\right)^{\perp}$, one concludes that $\left(\Phi^{*}\right)_{s} f-T_{\mathfrak{L} e}^{* *} f=0$. Therefore $\left(\Phi^{*}\right)_{s}\left(\operatorname{dom}\left(\left(\Phi^{*}\right)_{s}\right)^{*}\left(\Phi^{*}\right)_{s}\right) \subset \operatorname{ran} T_{\mathfrak{L} e}^{* *} \subset \mathfrak{E}$. Since $\operatorname{dom}\left(\left(\Phi^{*}\right)_{s}\right)^{*}\left(\Phi^{*}\right)_{s}$ is a core for the corresponding closed form, or equivalently, the closure of $\left(\Phi^{*}\right)_{s}\left\lceil\operatorname{dom}\left(\left(\Phi^{*}\right)_{s}\right)^{*}\left(\Phi^{*}\right)_{s}\right.$ is equal to $\left(\Phi^{*}\right)_{s}$, the claim follows:

$$
\mathfrak{F}=\overline{\operatorname{ran}}\left(\Phi^{*}\right)_{s} \subset \mathfrak{E} .
$$

(ii) $\Rightarrow$ (i) Assume that $\mathfrak{E}=\mathfrak{F}$. Then $\operatorname{ran}\left(\Phi^{*}\right)_{s}=\mathfrak{F}_{0} \subset \mathfrak{E}$ and $\left(\Phi^{*}\right)_{s} \subset \mathfrak{H} \times \mathfrak{E}$; see (3.5). Denote by $\left(\Phi^{*}\right)_{s e}$ the operator $\left(\Phi^{*}\right)_{s}$ viewed as an operator from $\mathfrak{H}$ to $\mathfrak{E}$. Then $\left(\Phi^{*}\right)_{s e}=P_{\mathfrak{E}}\left(\Phi^{*}\right)_{s} \subset P_{\mathfrak{E}} K^{*}=K_{e}^{*}$, so that $\left(\Phi^{*}\right)_{s e}=T_{\mathfrak{L}}$ with

$$
\mathfrak{L}=\operatorname{dom}\left(\Phi^{*}\right)_{s e}=\operatorname{dom} A^{\frac{1}{2}} \cap \operatorname{dom} B^{\frac{1}{2}} .
$$

Hence,

$$
\Phi^{* *} \Phi^{*}=\left(\left(\Phi^{*}\right)_{s}\right)^{*}\left(\Phi^{*}\right)_{s}=\left(\left(\Phi^{*}\right)_{s e}\right)^{*}\left(\Phi^{*}\right)_{s e}=T_{\mathfrak{L}}^{*} T_{\mathfrak{L}}^{* *},
$$

which shows that $\Phi^{* *} \Phi^{*}$ is extremal; cf. Theorem 3.4

As to the last statement, note that $P_{\mathfrak{E}}\left(\Phi^{*}\right)_{s} \subset P_{\mathfrak{E}} K^{*}=K_{e}^{*}$. Since $P_{\mathfrak{E}}\left(\Phi^{*}\right)_{s}=$ $P_{\mathfrak{E}} \Phi^{*}$ and hence $\left(\left(\Phi^{*}\right)_{s}\right)^{*} P_{\mathfrak{E}}=\Phi^{* *} P_{\mathfrak{E}}$, the selfadjoint relation $\Phi^{* *} P_{\mathfrak{E}}\left(P_{\mathfrak{E}} \Phi^{*}\right)^{* *}=$ $\left(\Phi^{* *} P_{\mathfrak{E}} \Phi^{*}\right)_{F}$ is an extremal extension of $A+B$ by Theorem 3.4

The nonnegative selfadjoint relation $\Phi^{* *} \Phi^{*}$ is called the form sum extension of the nonnegative relation $A+B$ (induced by the form (4.1)). Its multi-valued part is given by $\operatorname{mul} \Phi^{* *} \Phi^{*}=\operatorname{mul} \Phi^{* *}=\left(\operatorname{dom} \Phi^{*}\right)^{\perp}=\left(\operatorname{dom} A^{\frac{1}{2}} \cap \operatorname{dom} B^{\frac{1}{2}}\right)^{\perp}$. The orthogonal operator part of $\Phi^{* *} \Phi^{*}$ is the nonnegative selfadjoint operator which corresponds to the form sum (4.1) restricted to the closure of $\operatorname{dom} A^{\frac{1}{2}} \cap \operatorname{dom} B^{\frac{1}{2}}$. Theorem 4.1 indicates that the extremality of the form sum extension depends on the relation between the sets $\operatorname{dom} A \cap \operatorname{dom} B$ and $\operatorname{dom} A^{\frac{1}{2}} \cap \operatorname{dom} B^{\frac{1}{2}}$; cf. also Example 5.2 below. It also shows how to construct an extremal extension of $A+B$ from the form sum extension. 


\section{EXAmples}

Example 5.1. In the Hilbert space $\mathfrak{H}=L^{2}(1, \infty) \oplus L^{2}(1, \infty)$ consider the multiplication operators

$$
A=\left(\begin{array}{cc}
1 & t \\
t & t^{2}
\end{array}\right), \quad B=\left(\begin{array}{cc}
1 & -t \\
-t & t^{2}
\end{array}\right)
$$

each defined on its maximal domain. Note that the operators $A$ and $B$ are nonnegative and selfadjoint. The sum $A+B$ is given by the multiplication operator

$$
\left(\begin{array}{cc}
2 & 0 \\
0 & 2 t^{2}
\end{array}\right)
$$

on the domain $\operatorname{dom} A \cap \operatorname{dom} B$. The function $f(t)=\left(\begin{array}{ll}1 / t & 0\end{array}\right)^{\top} \in \mathfrak{H}$ does not belong to either $\operatorname{dom} A$ or to $\operatorname{dom} B$. However for each $n \in \mathbb{N}$ the function $f_{n}=\chi_{(1, n)} f$ belongs to $\operatorname{dom} A$ and to $\operatorname{dom} B$, and $f_{n} \rightarrow 2 f$ and $(A+B) f_{n} \rightarrow 2 f$ in $\mathfrak{H}$ as $n \rightarrow \infty$. Therefore the operator $A+B$ is not closed. However, the operator $A+B$ defined on $\operatorname{dom} A \cap \operatorname{dom} B$ has a natural selfadjoint operator extension given by (5.1) defined on its natural domain. In fact, it is not difficult to see that $A+B$ is essentially selfadjoint, so that there is precisely one selfadjoint extension of $A+B$ which is automatically nonnegative. Hence, the Krel̆n-von Neumann extension, the Friedrichs extension, and the form sum extension coincide. This example is due to Manfred Möller.

Observe that there exist nonnegative selfadjoint operators $A$ and $B$ for which

$$
\operatorname{dom} A \cap \operatorname{dom} B=\{0\}, \quad \operatorname{dom} A^{\frac{1}{2}} \cap \operatorname{dom} B^{\frac{1}{2}} \quad \text { dense in } \mathfrak{H} .
$$

It suffices to recall the existence of bounded nonnegative operators $C$ and $D$ for which $\operatorname{ran} C \cap \operatorname{ran} D=\{0\}, \operatorname{ran} C^{\frac{1}{2}} \cap \operatorname{ran} D^{\frac{1}{2}}$ dense in $\mathfrak{H}$; cf. [6]. Then $\operatorname{ker} C=$ ker $D=\{0\}$ and one can take $A=C^{-1}$ and $B=D^{-1}$.

Example 5.2. Let $A$ and $B$ be nonnegative selfadjoint relations in a Hilbert space $\mathfrak{H}$, which satisfy $\operatorname{dom} A \cap \operatorname{dom} B=\{0\}$. Then it is easily seen that the sum $S=A+B$ is a purely multi-valued relation $S=\{0\} \times \operatorname{mul}(A+B)$. The Krel̆n-von Neumann extension $S_{N}$ and the Friedrichs extension $S_{F}$ are given by $S_{N}=\mathfrak{K}^{\perp} \times \mathfrak{K}$ and $S_{F}=\{0\} \times \mathfrak{H}$, where $\mathfrak{K}$ is the closure of $\operatorname{mul}(A+B)$. The associated form $\mathfrak{t}_{N}$ is the null form on $\mathfrak{K}^{\perp}$, and the associated form $\mathfrak{t}_{F}$ is the null form on $\{0\}$. There exists a one-to-one correspondence between the set of all extremal extensions $\widetilde{A}$ of $S$ and the set of all closed subspaces $\mathfrak{L}$ of $\mathfrak{K}^{\perp}$; cf. (2.14) and Corollary 2.4. This correspondence is given by $\widetilde{A}=\mathfrak{L} \times \mathfrak{L}^{\perp}$, and the associated form $\widetilde{\mathfrak{t}}$ is the null form on $\mathfrak{L}$. If, in particular, $A$ and $B$ are nonnegative selfadjoint operators with $\operatorname{dom} A \cap \operatorname{dom} B=\{0\}$, then $\operatorname{mul}(A+B)=\{0\}, S=\{0,0\}$, and the form $\mathfrak{t}_{N}$ is the null form on $\mathfrak{H}$. Any extremal extension has a corresponding form which must be the null form on its domain. However, if $\operatorname{dom} A^{\frac{1}{2}} \cap \operatorname{dom} B^{\frac{1}{2}} \neq\{0\}$, then the form sum is nonzero on its domain, since $\operatorname{ker} A^{\frac{1}{2}} \cap \operatorname{ker} B^{\frac{1}{2}} \subset \operatorname{dom} A \cap \operatorname{dom} B=\{0\}$. Therefore, in this case the form sum is extremal only if $\operatorname{dom} A^{\frac{1}{2}} \cap \operatorname{dom} B^{\frac{1}{2}}=\{0\}$ and then it coincides with the Friedrichs extension. Finally, observe that if $\operatorname{dom} A^{\frac{1}{2}} \cap \operatorname{dom} B^{\frac{1}{2}} \neq$ $\{0\}$, then the form corresponding to $\Phi^{* *} P_{\mathfrak{E}}\left(P_{\mathfrak{E}} \Phi^{*}\right)^{* *}=\left(\Phi^{* *} P_{\mathfrak{E}} \Phi^{*}\right)_{F}$ in Theorem 4.1 is the null form on the closure of $\operatorname{dom} A^{\frac{1}{2}} \cap \operatorname{dom} B^{\frac{1}{2}}$. 


\section{REFERENCES}

[1] Yu.M. Arlinskiı̌, S. Hassi, Z. Sebestyén, and H.S.V. de Snoo, "On the class of extremal extensions of a nonnegative operator", Oper. Theory: Adv. Appl. (B. Sz.-Nagy memorial volume), 127 (2001), 41-81. MR.1902794(2003d:47028)

[2] Yu.M. Arlinskiı̌ and E.R. Tsekanovskiǔ, "Quasi selfadjoint contractive extensions of Hermitian contractions", Teor. Funkts., Funkts. Anal. Prilozhen, 50 (1988), 9-16. MR0975668 (90b:47014)

[3] E.A. Coddington, Extension theory of formally normal and symmetric subspaces, Mem. Amer. Math. Soc., 134, 1973. MR0477855 (57:17357)

[4] B. Farkas and M. Matolcsi, "Commutation properties of the form sum of positive, symmetric operators", Acta Sci. Math. (Szeged), 6 (2001), 777-790. MR1876466 (2003a:47050)

[5] B. Farkas and M. Matolcsi, "Positive forms on Banach spaces", Acta Math. Hungar., 99 (2003), 43-55. MR.1973084(2004d:47004)

[6] P.A. Fillmore and J.P. Williams, "On operator ranges", Adv. Math., 7 (1971), 254-281. MR0293441 (45:2518)

[7] S. Hassi, M. M. Malamud, and H. S. V. de Snoo, "On Kreĭn's extension theory of nonnegative operators", Math. Nachr., 274/275 (2004), 40-73.

[8] S. Hassi, A. Sandovici, H.S.V. de Snoo, and H. Winkler, "Form sums of nonnegative selfadjoint operators", Acta Math. Hungar., 111 (2006), 81-105. MR2188974

[9] S. Hassi, A. Sandovici, H.S.V. de Snoo, and H. Winkler, "A general factorization approach to the extension theory of nonnegative operators and relations", J. Operator Theory, to appear.

[10] Z. Sebestyén and J. Stochel, "Restrictions of positive self-adjoint operators", Acta Sci. Math. (Szeged), 55 (1991), 149-154. MR.1124953(92i:47024)

[11] Z. Sebestyén and J. Stochel, "On products of unbounded operators", Acta Math. Hungar., 100 (1-2) (2003), 105-129. MR1984863 (2004c:47003)

Department of Mathematics and Statistics, University of Vaasa, P.O. Box 700, 65101 VAASA, FinLAND

E-mail address: sha@uwasa.fi

Colegiul Naţional "Petru Rareģ", 610101, Str. Ştefan cel Mare, Nr. 4, Piatra Neamt, Romania

E-mail address: adrian.sandovici@yahoo.com

Department of Mathematics and Computing Science, University of Groningen, P.O. Box 800, 9700 AV Groningen, Nederland

E-mail address: desnoo@math.rug.nl

Institut für Mathematik, MA 6-4, Technische Universität Berlin, Strasse des 17. Juni 136, 10623 Berlin, Deutschland

E-mail address: winkler@math.tu-berlin.de 